\section{The Cytokeratins as Molecular Markers in Differential Diagnosis of Gastric Intestinal Metaplasia}

\author{
Vernygorodskyi Sergii* \\ Pathological Anatomy, Forensic Medicine and Law Department, Vinnitsa
} National Pirogov Memorial, Medical University, Vinnitsa, Ukraine

\begin{abstract}
The features of Cytokeratins (CK) expression in 336 patients with chronic atrophic gastritis and gastric intestinal metaplasia were studied on the basis of immunohistochemical analysis. A positive expression of CK7 in areas of intestinal metaplasia is an evidence of its immature, incomplete or gastrointestinal phenotype, but expression of CK20 is more distinctive for complete, mature/solely small-intestinal phenotype of gastric intestinal metaplasia.
\end{abstract}

Keywords: Cytokeratins; Intestinal Metaplasia; Stomach

Cytokeratins (CK) are subunits of epithelial cell intermediate filaments, which are genetically determined. There is some restriction of the types of cytokeratins expressed in different epithelium. Cytokeratin 7 (CK7) is a $54 \mathrm{kd}$ polypeptide found in normal epithelium ranging from lung, cervix, breast, and bile ducts to bladder transitional cell epithelium; however, it is essentially absent from gastrointestinal epithelia and squamous cell epithelium $[1,2]$. CK7 is present in fetal, largely absent in normal adult, and transiently neoexpressed in metaplastic and neoplastic epithelial cells of the stomach according to previous observations [3,4]. CK7 neoexpression in the stomach could, hence, define a fetal-like, dedifferentiated, cellular phenotype during the development of metaplasia and neoplasia [3]. Cytokeratin 20 (CK20) is mainly expressed in the cytoplasm of epithelial cells in the small and large intestine and in Merkel cells of the skin. Aberrant expression of CK may be observed in metaplastic, dysplastic and malignant cells $[3,4]$.

Intestinal Metaplasia (IM) is considered to be the most relevant metaplastic change in gastritis, and an incomplete IM, characterized by sulfomucin secretion and classified as IM type III,

*Corresponding author: Vernygorodskyi Sergii, Pathological Anatomy, Forensic Medicine and Law Department, Vinnitsa National Pirogov Memorial, Medical University, Vinnitsa, Ukraine, Tel: +380 679565453; Fax: +380 432350563; E-mail: vernsot@rambler.ru

Citation: Sergii V (2015) The Cytokeratins as Molecular Markers in Differential Diagnosis of Gastric Intestinal Metaplasia. J Cytol Tissue Biol 2: 007.

Received: December 19, 2014; Accepted: January 27, 2015; Published: February 11, 2015 has been identified as a significant risk factor and potential precursor of the intestinal type cancer [5]. The role of IM in cancer development is also stressed by a rare detection of microsatellite instability, and mutations of p53 and APC genes in the intestinal metaplastic cells. However, the consideration of IM as a precursor lesion of gastric cancer has its limitations. Recently, metaplastic and neoplastic changes in the stomach have increasingly been assessed using immunohistochemisty [6,7]. Only antigens present in all types of intestinal metaplasia, however, were described in these studies. Reliable markers for the immunohistochemical distinction between types I to III have not yet been evaluated. The expression of CK20 and CK7 as the components of intermediate filaments in gastrointestinal epithelial cells was investigated in types I to III intestinal metaplasia especially with regard to the difficulty of gaining reliable markers for appropriate distinction.

To assess the peculiarities of the of cytokeratins expression in different types of the stomach mucosa intestinal metaplasia.

\section{Materials and Methods}

Gastric biopsy specimens from 336 patients (192 men, 144 women; age range $26-72$ years; median $52.96 \pm 1,13$ years) with chronic gastritis and intestinal metaplasia were analyzed (Table 1). At least four biopsy specimens from both the antrum and the body were studied from each patient.

\begin{tabular}{|l|c|c|l|l|}
\hline & & & \multicolumn{2}{|l|}{$\begin{array}{l}\text { Chronic atrophic gastritis with } \\
\text { intestinal metaplasia } \\
\mathrm{n}=130\end{array}$} \\
& $\begin{array}{l}\text { Normal gastric } \\
\text { mucosa } \\
\mathrm{n}=46\end{array}$ & $\begin{array}{l}\text { Chronic trophic } \\
\text { gastritis } \\
\mathrm{n}=160\end{array}$ & $\begin{array}{l}\text { Complete } \\
\text { (Type I) } \\
\mathrm{n}=62\end{array}$ & $\begin{array}{l}\text { Incomplete } \\
\text { (type II and } \\
\text { III) } \\
\mathrm{n}=68\end{array}$ \\
\hline $\begin{array}{l}\text { Mean age, } \\
\text { years }\end{array}$ & 46 & 54 & 52 & 50 \\
\hline Male/female & $27 / 19$ & $94 / 66$ & $32 / 30$ & $39 / 29$ \\
\hline $\begin{array}{l}\text { Helicobacter } \\
\text { pylori }\end{array}$ & - & 52 & 24 & 47 \\
\hline CK20 & - & - & +++ & $-/+$ \\
\hline CK7 & - & - & $-/+$ & +++ \\
\hline
\end{tabular}

Table 1: Clinicopathological characteristics of patients with chronic atrophic gastritis and intestinal metaplasia.

CK - Cytokeratins expression: - absent, + weak, +++ strong

Routine histology included haematoxylin and eosin staining as well as Giemsa staining. Gastritis was classified according to the Sydney classification system. In each case Helicobacter pylori colonization of the epithelium was confirmed. Specimens showing ulcers, dysplasia, or carcinoma were excluded from the study. Sections were further examined using the periodic acid Schiff reaction, and alcian blue, $\mathrm{pH} 2.5$, staining/high iron diamine staining. Our panel of primary monoclonal antibodies included: CK20 and CK7 (1:100; DAKO, Denmark). 


\section{Results}

In the groups of patients with normal gastric mucosa, with Chronic non Atrophic Gastritis and Atrophic Gastritis (CAG) without IM we did not observe CK20 expression in gastric epithelial cells. In immunohistochemical analysis of cases with complete IM, we have found a strong expression of CK20 in both columnar epithelial cells and goblet exocrinocytes, thus marking prevailed in the upper compartment of the gastric mucosa, such as in the surface epithelium of gastric foveolas (Figure 1). Our study found a more pronounced expression of CK20 in the case of I and III histochemical type of intestinal metaplasia and weak expression CK20 in the case of IM type II, indicating the immaturity of the last one. Immunoreactivity was restricted to the tips and the upper foveolae. In the deep foveolae positive staining was not seen. No differences were found between the antrum and the body. We observed the strongest expression of CK7 in areas of incomplete IM (Figure 2,3) and the epithelium of cystic dilated glands and small glands, which form the tubular structures ( $96 \%$ of patients with CAG with IM). In areas of complete IM CK7 expression was weak in $67 \%$ and in $33 \%$ of these patients was negative $(\mathrm{p}<0.05)$. However, over time (a period of 2 to 6 years) when changing / appearance of incomplete type IM in patients with CAG we noted enhance expression of CK7. Also, we have observed increasing of expression of CK7 in $57 \%$ H. pylori (+) patients with CAG with various types of IM, but the difference between them and $H$. pylori (-) patients was not reliable $(\mathrm{p}>0.05)$.

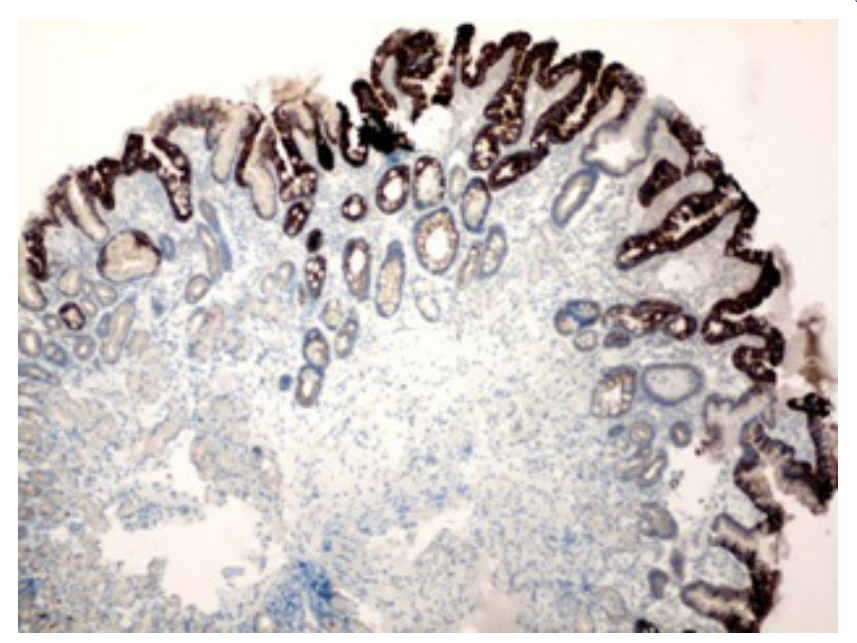

Figure 1: A strong expression of CK20 in columnar and goblet cells prevailed in the upper compartment of the gastric mucosa with complete intestinal metaplasia. Immunohistochemistry of anti-CK20, x 100.

\section{Discussion}

Gastric IM is similar histologically and immunohistochemically to Barrett's esophagus/esophageal adenocarcinoma. It is defined as the development of goblet and/or Paneth cells within the normal gastric mucosa [8]. The two main types of gastric IM are the complete type (type I) characterized by its resemblance to normal small intestinal mucosa with absorptive cells, Paneth cells and goblet cells; and the incomplete type (types II and III) where there are columnar and goblet cells. Most cases of gastric carcinoma arise within areas of incomplete gastric IM, and show CK7 and CK20 in the superficial and deep crypt cells $[9,10]$.

Differential patterns of cytokeratin expression have been demonstrated in the oesophagus and proposed as useful clinical

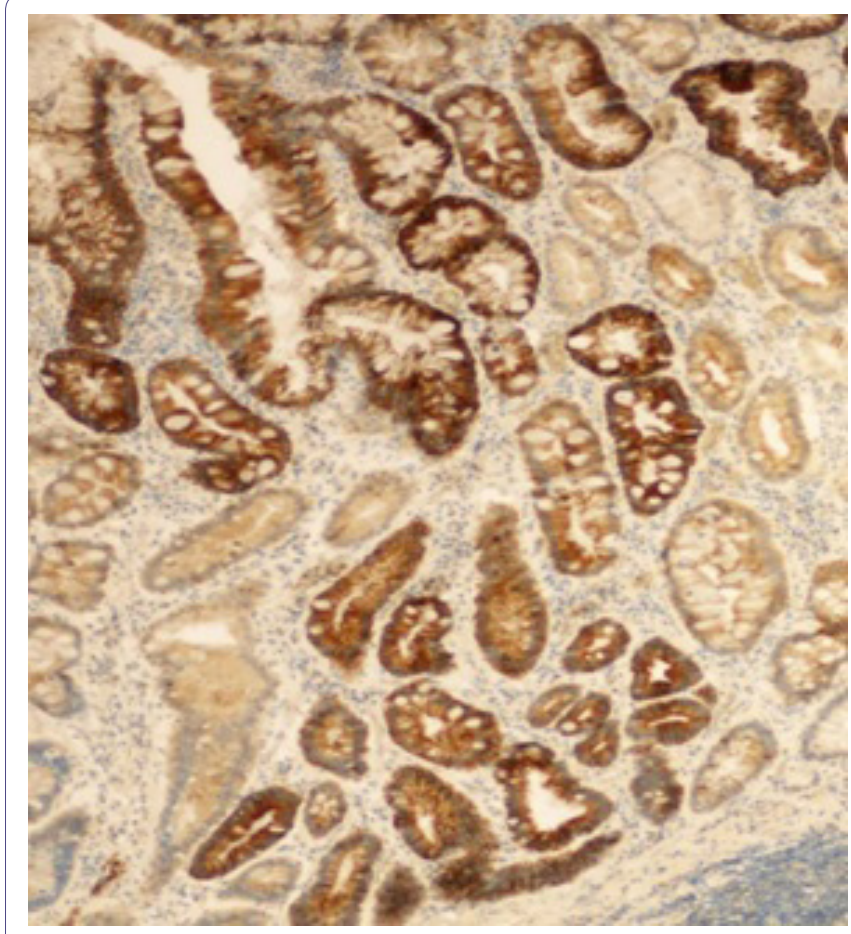

Figure 2: A strong expression of CK7 in columnar and goblet cells prevailed in the lower compartment of the gastric mucosa with incomplete intestinal metaplasia. Immunohistochemistry of anti-CK7, x 100.

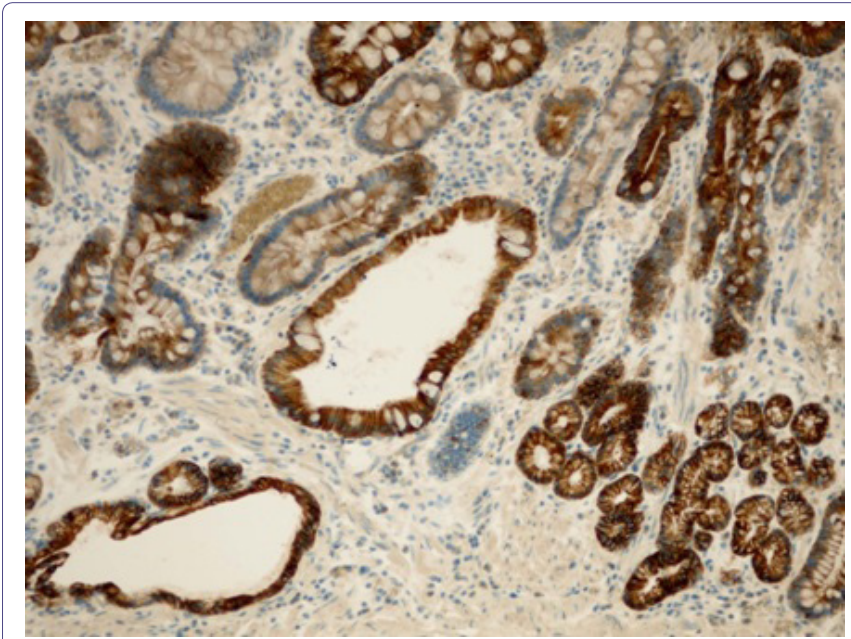

Figure 3: A strong expression of CK7 in areas of incomplete IM, in the epithelium of cystic dilated glands and small glands, which form the tubular structures. Immunohistochemistry of anti-CK7, x 200.

markers. However, a unique pattern of CK7 and CK20 immunohistochemical expression, designated the BM CK7/20 pattern, has been demonstrated to be both sensitive and specific to Barrett's oesphagus and may be used as an objective marker of Barrett's Metaplasia (BM) [11,12]. This pattern shows superficial CK20 staining and strong CK7 staining of both superficial and deep glands and can be used to distinguish BM from intestinal metaplasia of the stomach which, although can be histologically indistinguishable, has a different pattern of cytokeratin expression, is associated with Helicobacter pylori infection, and is not associated with an increased 
risk of malignancy $[13,14]$. However, the specificity of the cytokeratins were controversial and some recent studies have raised the question as to its specificity $[10,14]$.

Thus in our investigation a positive expression of CK7 in areas of intestinal metaplasia is an evidence of its immature, incomplete or gastrointestinal phenotype, but expression of CK20 is more distinctive for complete, mature/solely small-intestinal phenotype of gastric intestinal metaplasia.

In conclusion, greater understanding of the processes involved in the development and progression of IM is crucial if we are to develop strategies to intervene at an earlier stage in the metaplasia-dysplasia-carcinoma sequence. Perhaps it is in the understanding of the biology of IM where CK have already made a potentially useful contribution. Cytokeratins are very stable proteins and are conserved in most epithelial cancers, even in the presence of gross phenotypic and genetic alterations. For this reason, detection of CK subsets such as CK7 and CK20 have become very useful in diagnosing the origins of occult or poorly differentiated cancers and are part of current clinical practice.

\section{References}

1. Salo JA, Kivilaakso EO, Kiviluoto TA, Virtanen IO (1996) Cytokeratin profile suggests metaplastic epithelial transformation in Barrett's oesophagus. Ann Med 28: 305-309.

2. Moll $R$ (1998) Cytokeratins as markers of differentiation in the diagnosis of epithelial tumors. Subcellular Biochemistry. In: Hermann H, Harris JR (eds.). Intermediate Filaments. Plenum Press, New York, USA 31: 205-262.

3. Nobusawa A, Sano T, Negishi A, Yokoo S, Oyama T (2014) Immunohistochemical staining patterns of cytokeratins 13,14 , and 17 in oral epithelial dysplasia including orthokeratotic dysplasia. Pathol Int 64: 20-27.

4. Kirchner T, Müller S, Hattori T, Mukaisyo K, Papadopoulos T, et al. (2006) Metaplasia, intraepithelial neoplasia and early cancer of the stomach are related to dedifferentiated epithelial cells defined by cytokeratin-7 expression in gastritis. Virchows Arch 439: 512-522.
5. Niwa T, Ikehara Y, Nakanishi H, Tanaka H, Inada K, et al. (2005) Mixed gastric- and intestinal-type metaplasia is formed by cells with dual intestinal and gastric differentiation. J Histochem Cytochem 53: 75-85.

6. Chan CW, Wong NA, Liu Y, Bicknell D, Turley H, et al. (2009) Gastrointestinal differentiation marker Cytokeratin 20 is regulated by homeobox gene CDX1. Proc Natl Acad Sci USA 106: 1936-1941.

7. Zheng Y, Wang L, Zhang JP, Yang JY, Zhao ZM, et al. (2010) Expression of p53, c-erbB-2 and Ki67 in intestinal metaplasia and gastric carcinoma. World J Gastroenterol 16: 339-344.

8. Wong HH, Chu P (2012) Immunohistochemical features of the gastrointestinal tract tumors. J Gastrointest Oncol 3: 262-284.

9. Jovanovic I, Tzardi M, Mouzas IA, Micev M, Pesko P, et al. (2002) Changing pattern of cytokeratin 7 and 20 expression from normal epithelium to intestinal metaplasia of the gastric mucosa and gastroesophageal junction. Histol Histopathol 17: 445-54.

10. Schilling D, Spiethoff A, Rosenbaum A, Hartmann D, Eickhoff A, et al. (2005) Does Cytokeratin7/20 immunoreactivity help to distinguish Barrett's esophagus from gastric intestinal metaplasia? Results of a prospective study of 75 patients. Pathol Res Pract 200: 801-805.

11. Ormsby AH, Goldblum JR, Rice TW, Richter JE, Falk GW, et al. (1999) Cytokeratin subsets can reliably distinguish Barrett's esophagus from intestinal metaplasia of the stomach. Hum Pathol 30: 288-294.

12. Liu GS, Gong J, Cheng P, Zhang J, Chang Y, et al. (2005) Distinction between short-segment Barrett's esophageal and cardiac intestinal metaplasia. World J Gastroenterol 11: 6360-6365.

13. Shearer C, Going J, Neilson L, Mackay C, Stuart RC (2005) Cytokeratin 7 and 20 expression in intestinal metaplasia of the distal oesophagus: relationship to gastro-oesophageal reflux disease. Histopathology 47: 268-275.

14. Mohammed IA, Streutker CJ, Riddell RH (2002) Utilization of cytokeratins 7 and 20 does not differentiate between Barrett's esophagus and gastric cardiac intestinal metaplasia. Mod Pathol 15: 611-616. 